\title{
Understanding orthopedic infections through a different perspective: Microcalorimetry growth curves
}

\author{
MIHNEA POPA $^{1,2}$, ADRIAN CURSARU $^{1,2}$, VLAD POPA $^{3}$, ALEXANDRU MUNTEANU $^{4,5}$, BOGDAN ȘERBAN $^{1,2}$, \\ BOGDAN CREȚU ${ }^{1,2}$, SERGIU IORDACHE ${ }^{1,2}$, CATALIN GABRIEL SMARANDACHE ${ }^{6,7}$, \\ CARMEN ORBAN $^{8}$ and CĂTĂLIN CÎRSTOIU ${ }^{1,2}$ \\ ${ }^{1}$ Department of Orthopedics and Traumatology, 'Carol Davila' University of Medicine and Pharmacy, 020021 Bucharest; \\ ${ }^{2}$ Department of Orthopedics and Traumatology, University Emergency Hospital, 050098 Bucharest; \\ ${ }^{3}$ 'Ilie Murgulescu' Institute of Physical Chemistry, 060021 Bucharest; ${ }^{4}$ Department of Medical Microbiology, \\ 'Carol Davila' University of Medicine and Pharmacy, 020021 Bucharest; ${ }^{5}$ Cantacuzino National Institute of Research and \\ Development for Microbiology and Immunology, 050096 Bucharest; ${ }^{6}$ Department of General Surgery, \\ 'Carol Davila' University of Medicine and Pharmacy, 020021 Bucharest; ${ }^{7}$ Department of General Surgery, \\ University Emergency Hospital, 050098 Bucharest; ${ }^{8}$ Department of Anaesthesia and Intensive Care, \\ 'Carol Davila' University of Medicine and Pharmacy, 020021 Bucharest, Romania
}

Received September 3, 2021; Accepted October 5, 2021

DOI: $10.3892 /$ etm.2022.11189

\begin{abstract}
Infectious disease is a serious healthcare problem in orthopedics, as well as other surgical specialties. Accurate and prompt diagnosis, as well as proper care, is critical, as infection of a surgical wound, particularly in the case of arthroplasties or the use of orthopedic implants, can have a catastrophic effect in most cases, necessitating the removal of foreign material. Lyophilized bacteria samples were obtained from Cantacuzino National Institute of Research and Development for Microbiology and Immunology and investigated microcalorimetrically. Isothermal microcalorimetry measures the temperature generated by the multiplication of microorganisms; using an adapted program, it describes the growth curve in real-time according to the received electrical signal. The thermograms of Escherichia coli and Klebsiella pneumonie were analyzed, and similarities were observed for both the time required for the bacteria to grow and the heat flow generated by their growth. Bacterial microcalorimetry has a variety of benefits, and should be regarded as a means of rapid and accurate diagnosis. Sensitivity is a valuable attribute for a diagnostic technique; when only a few microorganisms are present, microcalorimetric signs of bacterial multiplication can be observed. Microcalorimetry has potential as a simple diagnostic tool in a variety of infections, but further research
\end{abstract}

Correspondence to: Dr Adrian Cursaru, Department of Orthopedics and Traumatology, 'Carol Davila' University of Medicine and Pharmacy, 8 Bulevardul Eroii Sanitari, Sector 5, 020021 Bucharest, Romania

E-mail: cursaru_adrian@yahoo.com

Key words: microcalorimetry, orthopedics, infection is needed to ensure that it is used correctly. A thorough investigation (including kinetic analysis) of a reproducible thermal signal of bacterial growth could lead to the creation of new methods for quickly identifying bacteria.

\section{Introduction}

Infectious disease occurring during surgery is a serious healthcare problem; in orthopedics, as well as other surgical specialties, a wound infection causes the healing process to be delayed, as well as an increase in the clinical burden faced by the patient. Patients in these cases report greater suffering than patients that were cured without infection while having the same pathology (1). Accurate and prompt diagnosis, as well as proper care, is critical, because infection of a surgical wound, particularly in the case of arthroplasties or the use of orthopedic implants, can have a catastrophic effect in most cases, necessitating the removal of foreign material $(2,3)$.

According to the literature, an exponential rise in arthroplasty procedures for maintaining or improving mobility have been accompanied with an increase in life expectancy (4). Owing to an increase in the amount of traffic accidents and sports injuries, the use of joint replacements and orthopedic implants is growing (5). Biocompatible prosthetics are used in the treatment of degenerative pathologies, as well as for cosmetic purposes $(6,7)$.

When a foreign body is present, the risk of infection is substantially higher; nearly half of all nosocomial infections are linked to medical equipment in some way $(8,9)$. Infection can originate from an infected implant, as well as disinfection and sterilization errors. For these reasons, any infectious outbreaks must be investigated, located and treated in the case of suspicion of postoperative septic evolution (10). Orthopedic infections are more common in patients with dental abscesses, skin or digestive infections (11). Staphylococcus aureus 
(S. aureus), coagulase-negative staphylococci, Escherichia coli (E. coli), Enterobacter spp., Klebsiella pneumonie (K. pneumonie). and Pseudomonas aeruginosa are among the bacteria commonly present; $S$. aureus is the most frequently detected of these (11-13). Factors that can reduce the risk of infection include the introduction of flow restriction in the operation room, highly qualified surgeons with reduced operation time, the application of prophylactic antibiotics and, when applicable, the use of antibiotic-charged implants $(14,15)$.

A technique that has found a place in the ever-changing context of technological evolution is microcalorimetry. This technique, widely employed in inorganic chemistry, is only starting to find more widespread use in medicine. The basis of microcalorimetry is the capacity of bacteria to produce heat from metabolic activity; a microcalorimeter records the energy released due to different biochemical reactions, which can be used to generate growth curves for clinical evaluation (16-20).

Infectious pathology in the field of orthopedics and traumatology occurs less frequently than in other medical or medicosurgical specialties (21); however, musculoskeletal infections can lead to negative outcomes, and are relatively difficult to identify and treat. Making a positive diagnosis and evaluating the options based on the diagnosis, as well as treating the pathology as soon as possible, are critical.

An unidentified infection can impair the functioning of a limb or, if it leads to sepsis, can cause life-threatening circumstances $(22,23)$. Osteomyelitis is a disease that can affect individuals of all ages and involve any part of the skeletal system; it can and should be categorized based on the type of host response, the length of the infection or the age of the infected individual in order to select the most optimal therapeutic approach and determine the prognosis (24). Concerning onset time, osteomyelitis may be categorized as acute if it occurs within the first 6 weeks of infection, or chronic if the onset occurs after 6 weeks. At this stage, localized infection can occur in one of two ways $(23,24)$. When a microorganism penetrates from the outside into the bone, it is known as direct inoculation (following trauma, during surgery or when a neglected septic process develops in the vicinity of the bone); alternatively, remote inoculation results from a distant outbreak area that involves the bone via hematogenous dissemination of the pathogen $(24,25)$. These cases are frequently plurimicrobial infections, which affect easily identifiable anatomical areas and, in the case of trauma, are followed by a major, quantitative contamination of the wound $(23,25)$.

Infection involves a compromised local environment with devitalized tissues and necrosis, both of which are conducive to the development of septic processes due to suboptimal vascularization that prevents or impairs penetration of antimicrobial substances $(22,24,25)$. Following the development of a distant septic process, hematogenous dissemination of microbial agents occurs, resulting in bacteremia. During bacteremia, infections of the synovial fluid, cartilage or bones are possible. Microorganisms are typically restricted to sites of damage, such as a fracture treated with osteosynthesis or a joint prosthesis. Diabetes or smoking, which affect the structures of small vessels, as well as liver failure, kidney failure and conditions involving immunodeficiency, are all host-related systemic factors that may promote the spread of infection $(22,24)$. In these cases, the patient's age is critical, as infections resulting from this process are heavily affected by local anatomy and physiology $(23,25,26)$.

To understand the basics, it is necessary to discuss other technical concepts. Preliminary principles of thermodynamics discovered by Thomas Johann Seebeck (late 18th century) are often referred to as the Seebeck effect. Different materials with the same mass may require different levels of heat to raise their temperature by $1^{\circ} \mathrm{C}(27,28)$. The Seebeck effect (direct thermoelectric effect) describes the generation of thermoelectromechanical tensions in a circuit, which are converted into a temperature difference between two materials with different structures $(27,28)$.

Peltier's effect is the second physical fundamental; it should be considered an inversion of the previously discussed effect, as it describes a process in which a potential difference is converted into a temperature difference $(27,28)$. This effect, which can be seen in thermoelectric heating and cooling systems, was discovered in 1834 by French physicist Jean Charles Athanase Peltier, who demonstrated through experiments that if an electric current passes through a junction of two different metals, it can register losses or increases in temperature at that zone. The value of absorbed warmth is equal to the value of emitted warmth, based on Peltier's coefficient $(27,28)$.

Modern microcalorimetry dates back to the 19th century, when physicist Albert Tian built a calorimeter (depth, $7 \mathrm{~m}$ ) in order to maintain stable temperatures. Peltier and Seebeck effects are at the heart of microcalorimeter activity, both in the past and in the present, resulting in two of the most critical components: The Seebeck effect, which is used to create a temperature sensor that can convert a temperature difference into an electric signal; by means of a Peltier-type cell, the applied electrical energy is transformed in absorbed or released heat (28).

Tian succeeded in studying insect metabolism using a microcalorimeter and the Peltier effect in 1922, and Edouard Calvet, his successor, succeeded in transforming the microcalorimeter into a laboratory apparatus in 1948 by developing two twin calorimetric components: The reference and the sample $(28,29)$. The first microcalorimeter was sold in 1970 (29), and it has evolved since then due to technological advancements. Microcalorimeters have been developed with increasing sensitivity, the ability to acquire a greater quantity of data due to the availability of multiple channels and computerized technology that can process a wide variety of data in a short amount of time $(30,31)$. The present study aimed to demonstrate that this tool could be used to diagnose infectious pathologies in the field of orthopedics by evaluating different bacterial strains using the method.

\section{Materials and methods}

\section{Theoretical considerations}

Thermal analysis. Microcalorimetry is a science that uses a calorimeter to measure the heat generated by different processes. This process is called heat flux, and it is based on the physical principle that when two bodies with different temperatures are brought together, they will adjust their temperatures until they reach a thermal equilibrium (32). To record this occurrence, calorimeters will measure temperatures and collect data in an indirect manner, as thermal changes will be converted into electric signals, which will be 
recorded and processed through an informatics program that will allow data interpretation (32).

In order to conduct a thermal analysis, some temperature changes must occur, which must be recorded; in the present study, a differential measurement method has been used. For these experiments, changes in temperature that occur in one probe compared to a reference when the temperature is changed are used; for an experiment to be successful, one property must be different between the two cells (which house the probe and the reference) in order to properly quantify and analyze the results. It should be noted that this property can be changed in subsequent experiments to evaluate various changes. The calorimeter has two rooms that are equipped with temperature sensors; following the introduction of the two cells (sample and reference), they regulate the flow of heat between the two cells, as well as between cells and structures in the body.

When it comes to twin cells (which, as previously stated, have similar properties), the amount of energy transferred into the cell has no effect; only the difference between the two is recorded, which is known as differential type microcalorimetry $(33,34)$.

Role of microcalorimetry in bacteriological research. Numerous studies have been conducted using microcalorimetry, investigating bacterial metabolism and how bacteria react to changes in the culture environment, including the levels of nutritive substances, temperature or the amount of oxygen in the microcalorimetric cells $(31,34)$. All of these variables are necessary and must be factored into the equation, as changes in their order can have a substantial impact on calorimetric detection and the rate of bacterial growth. The majority of studies are conducted in closed systems, which contain a substance in a finite quantity, allowing bacterial culture to represent a finite growth cycle that can be quantified.

A bacterial culture has four stages of growth (Fig. 1) $(35,36)$ : i) Lag phase/latency phase, during which the bacteria adapt to the environment in which they were inoculated, with protein synthesis and low-intensity metabolic reactions, but no division; ii) exponential growth phase, during which bacteria grow as long as environmental conditions are favorable (the bacterial growth rate can be calculated using an exponential function with base $2, \mathrm{X}=\mathrm{X}_{0} 2^{\mathrm{n}}$, as they multiply via binary division; this evolution can also be expressed as a function of time, as the generation time is represented by the interval between two successive multiplications); iii) stationary phase, during which the rate of bacterial generation becomes equal to that of bacterial death due to the increase in the quantity of inhibitors and decrease in substances necessary for growth; and iv) decline phase, at the end of which most bacteria are destroyed as a result of the conditions in the stationary phase, leaving a low number of bacteria in a non-multiplicative state that can resume the cycle if they are offered favorable environment (37).

Demonstrating the variability and reproducibility of the process using American type culture collection (ATCC) reference strains. To conduct superimposable experiments, the phases of experimental planning must be conducted in the same manner with each experimental repeat. Preparing a liquid culture of the infectious agent to be studied is the first step in conducting an experiment. As the amount of non-viable bacteria accumulates in the investigated environment over time, an elevated artificial nephelometric index may be misleading; thus, cultures must be freshly prepared for each experiment, as the bacteria used have the capacity to grow at room temperature $(13,19,21,32)$. Therefore, the second important step is to prepare the samples and insert them into the microcalorimeter as soon as possible. When an experiment is performed after a long period of time has elapsed since the sample was prepared, the initial growth stage may be lost from the recording timeline.

Another critical consideration when using a microcalorimeter is maintaining a stable ambient temperature, as changes in the room can create artifacts in the bacterial growth curve. Considering the above, it may be assumed that establishing a working procedure and standard rules of conduct for microcalorimetry is crucial for efficient experiments.

The following microorganisms were used for the experiments intended to establish reproducibility: E. coli (ATCC 25922) and K. pneumonie (ATCC 700603), which were accessed with the goodwill of the medical staff at the Cantacuzino National Institute of Research and Development for Microbiology and Immunology. The bacteria were grown on trypticase soy agar [TSB; mixture of pancreatic digest of casein $(17 \mathrm{~g}), \mathrm{NaCl}(5 \mathrm{~g})$, papaic digest of soybean meal $\left.(3 \mathrm{~g}), \mathrm{K}_{2} \mathrm{HPO}_{4}\right)$ $(2.5 \mathrm{~g})$ and dextrose $(1.8 \mathrm{~g})$, diluted to 1 liter and $\mathrm{pH} 7.3 \pm 0.2$ at $25^{\circ} \mathrm{C}$ ] and Sabouraud dextrose agar [SDA; mixture of mycological peptone $(10 \mathrm{~g})$, dextrose $(40 \mathrm{~g})$ and agar $(15 \mathrm{~g})$, diluted to 1 liter and $\mathrm{pH} 5.6$ at $25^{\circ} \mathrm{C}$ ], which were composed in-house.

Procedure. Experiments were conducted as follows. First, $3,000 \mu \mathrm{l}$ of sterile medium (SDA or TSB, depending on the experiment) was added to a nephelometric tube, and the McFarland index was measured using a nephelometer. Then, $15 \mu \mathrm{l}$ pathological produce was dispersed in $300 \mu \mathrm{l}$ medium, ensuring that the microorganisms were homogeneously dispersed. Then, $2 \mu \mathrm{l}$ solution was repeatedly pipetted in the nephelometric tube until the McFarland index rose by 1.0. Sample cells were filled with $600 \mu \mathrm{l}$ inoculated medium at room temperature and hermetically sealed using a silicon o-ring. A batch cell containing $600 \mu 1$ sterile TSB or SDA was used as the reference for differential scanning microcalorimetry. Both cells were sealed and then inserted into the microcalorimeter.

After both the sample and the reference cell were inserted, the acquisition program was initiated and set to maintain a temperature of $37^{\circ} \mathrm{C}$. The recording was terminated and analyzed after the curve reached the isoelectric line for 2-3 h, which is the point where the bacterial culture no longer produced energy. Only experiments conducted at $37^{\circ} \mathrm{C}$ with a loading volume of $600 \mu \mathrm{l}$ were included in the present study.

The tracking of bacterial growth curves and the primary processing of data obtained by the microcalorimeter was performed using Calisto (v1.493; Setaram; KEP Technologies), and R (v3.5.0) (38) and RStudio server packages (v1.1.456) (39) were used for the processing, comparison and statistical analysis of data.

\section{Results}

In the present study, two separate experiments were conducted with each bacterial strain, K. pneumonie (Fig. 2) and E. coli 


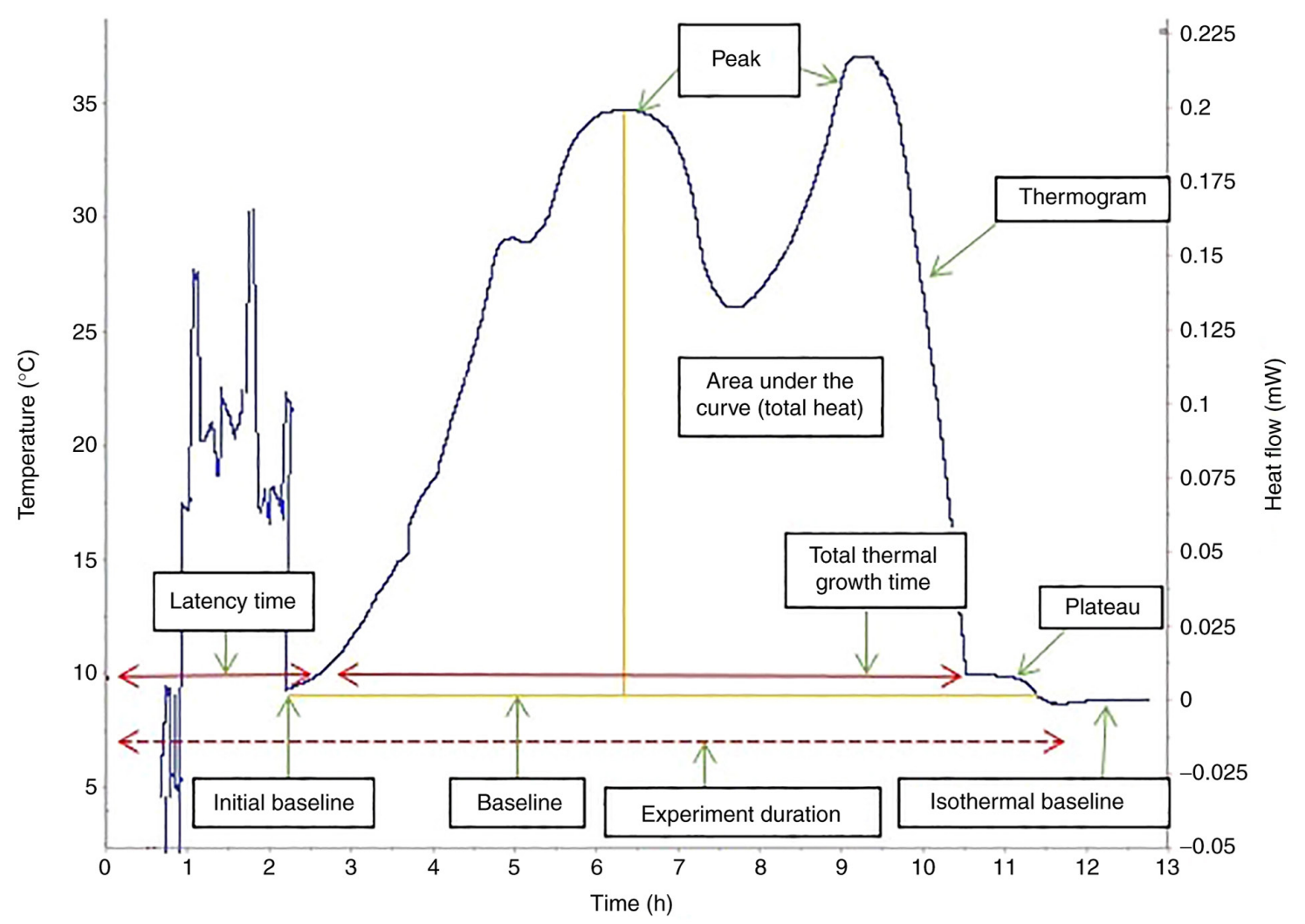

Figure 1. Recording of the differential scanning microcalorimetric signal. Recordings are presented as a graph of heat flux dependence (heat flow, mW) against time and temperature of the external source. In this graph, a positive heat flow represents an exothermic process.

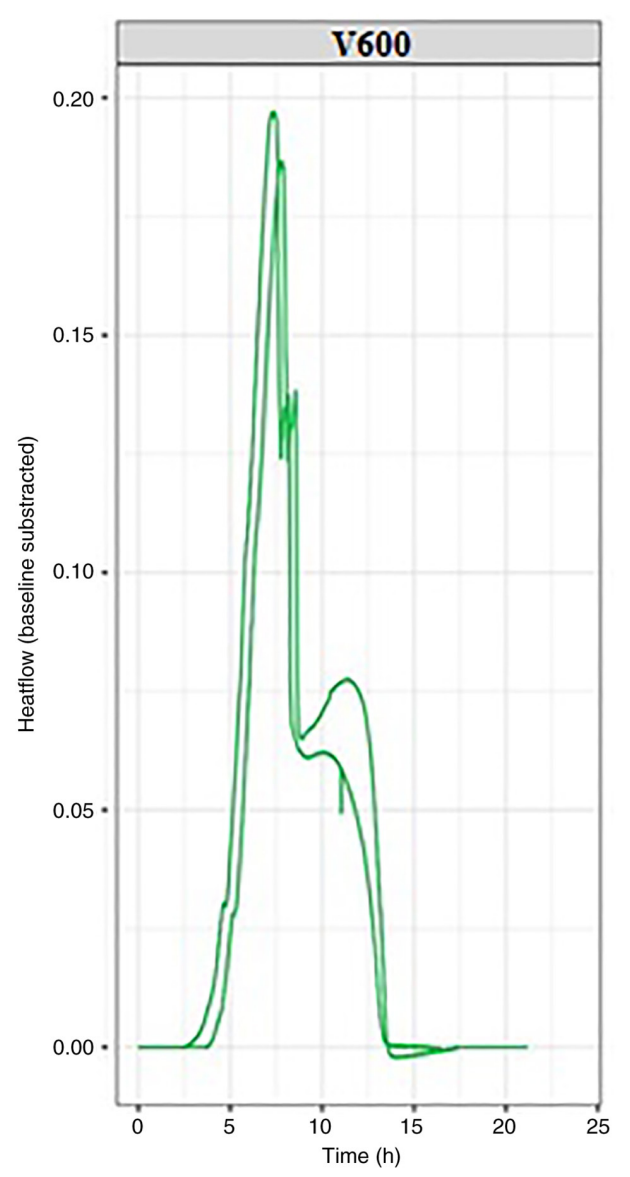

Figure 2. Thermogram of Klebsiella pneumoniae. Overlap of two experiments is presented.

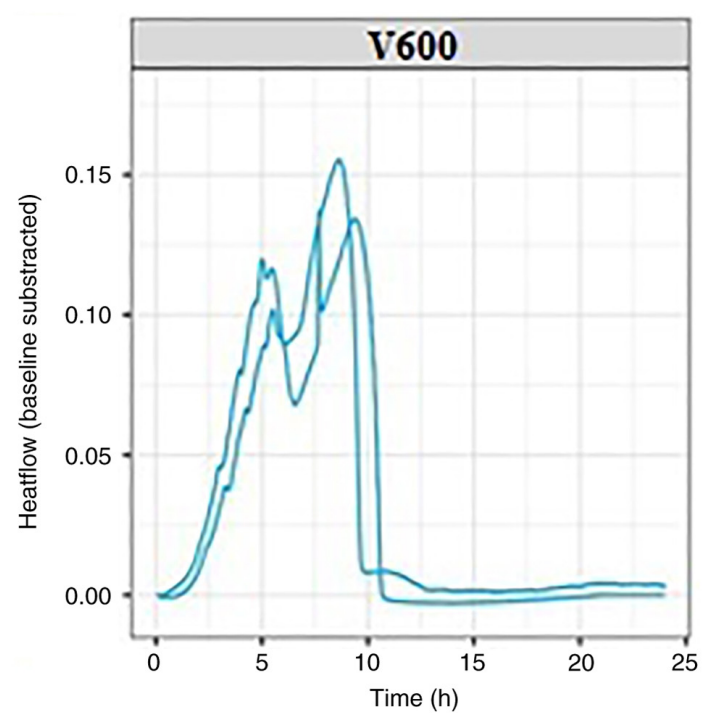

Figure 3. Thermogram of Escherichia coli. Overlap of two experiments is presented.

(Fig. 3); each experiment was conducted at $37^{\circ} \mathrm{C}$, the normal temperature at which the bacteria develop. The thermograms can be evaluated based on key points determined by a set of quantitative parameters, including thermal signal detection, establishment of the exponential growth, the peak maximum and the return to baseline (Fig. 1). Measurements determined based on these points (including the corresponding positions of, or intervals between events on the time scale, as well as heat 
flow values) can be used to characterize raw bacterial growth thermograms, as well as to differentiate the microorganisms.

By evaluating the thermograms for both $E$. coli and $K$. pneumonie, similarities were observed for both experimental repeats for each bacterial strain, including in the time required for bacterial growth and the heat flow generated by their growth. The bacterial growth and imprint unique to each strain used in the experiment can be followed in the graphs, which can be viewed in real-time. For each bacterial strain, the growth curves reached the isoelectric line within 10-15 h from the start of the experiment.

\section{Discussion}

Bacterial microcalorimetry has a variety of benefits, and should be regarded as a means of rapid and accurate diagnosis. Sensitivity is a valuable attribute; when only a few microorganisms are present, microcalorimetric signs of bacterial multiplication can be observed $(40,41)$. The generated curve has features that help identify the pathogen involved, in addition to demonstrating the presence of microorganisms. It is worth noting that this approach provides real-time data, the progression of bacterial growth can be tracked at any time using standard laboratory equipment or portable devices. In addition to the efficiency with which data can be processed, a microcalorimetric experiment allows researchers to evaluate a greater number of variables (temperature, culture medium, recording method) that correspond with knowledge already available in the literature, providing greater options for diagnosis and targeted care $(33,34,40)$. Any approach that promotes targeted treatment should be sponsored and developed in a world where the threat of increasing microorganism resistance to antibiotics and chemotherapeutics is widely recognized. Within the context of orthopedics, the chronic pain caused by a pathology, whether surgically treated or not, will lower patient satisfaction, regardless of whether or not comorbidities are present. Inadequate or painful mobility can lead to depression and exacerbation of other pathologies, so effective treatment of infection should be considered in any situation $(10,24,42,43)$.

The microcalorimetric method of identifying bacteria based on their growth curves is still in progress, with no substantial body of research at present. The probability of widespread use should be considered, but research this stage will require extensive research, involving processing with specialized software in order to develop of a widely applicable algorithm. A thorough investigation (including kinetic analysis) of a reproducible thermal signal of bacterial growth could lead to the creation of new methods for quickly identifying bacteria. Our research is limited to a small field within infectious medical pathology, but it is hypothesized that microcalorimetry has the potential to be applied in a wide range of medical fields.

In the graphs presented in this article, two growth curves can be observed for each pathogen. Within these growth curves, the growth pattern is identical but minimal changes in the amount of energy released can be observed. These differences occur as experiments are performed successively without using the same culture medium prepared for the first experiment in the series, resulting in minute differences between experimental repeats; this will be addressed in future by purchasing newer generation devices, but it also emphasizes the sensitivity of the microcalorimetry method, which can detect otherwise imperceptible differences.

Wound infection and the release of pro-inflammatory modulators leads to local discomfort and delayed healing. Pain-related stress impairs the immune response to infection, further impairing wound healing $(44,45)$. With this in mind, the development of innovative and quick diagnostic methods is important for providing rapid care that will alleviate patient suffering, reduce hospitalization times and decrease the burden on the medical system.

\section{Acknowledgements}

Not applicable.

\section{Funding}

Not applicable.

\section{Availability of data and materials}

All data generated or analyzed during this study are included in this published article.

\section{Authors' contributions}

MP drafted the manuscript, and made substantial contributions to conception and design. BS and SI designed the study. AM and $\mathrm{BC}$ acquired data. AC analyzed data, whereas VP interpreted the data. CGS was involved in revising the manuscript and interpreted data. $\mathrm{CO}$ and $\mathrm{CC}$ provided final approval of the manuscript, as well as making substantial contributions to conception and design. All authors read and approved the final manuscript.

\section{Ethics approval and consent to participate}

Not applicable.

\section{Patient consent for publication}

Not applicable.

\section{Competing interests}

The authors declare that they have no competing interests.

\section{References}

1. Soon K and Acton C: Pain-induced stress: A barrier to wound healing. Wounds UK 2: 92-101, 2006.

2. Preoteasa CT, Preoteasa E, Popa M, Pircalabrioru Gradisteanu G, Grigore R and Arutescu L: In vitro characterization of microbial biofilm on soft materials used in overdentures retained by mini implants. Rom Biotechnol Lett 24: 10-19, 2019.

3. Varnum C, Pedersen AB, Rolfson O, Rogmark C, Furnes O, Hallan G, Mäkelä K, de Steiger R, Porter M and Overgaard S: Impact of hip arthroplasty registers on orthopaedic practice and perspectives for the future. EFORT Open Rev 4: 368-376, 2019. 
4. Conen A, Fux CA, Vajkoczy P and Trampuz A: Management of infections associated with neurosurgical implanted devices. Expert Rev Anti Infect Ther 15: 241-255, 2017.

5. Hexter AT, Hislop SM, Blunn GW and Liddle AD: The effect of bearing surface on risk of periprosthetic joint infection in total hip arthroplasty: A systematic review and meta-analysis. Bone Joint J 100-B: 134-142, 2018.

6. Jiang B, Liang S, Peng ZR, Cong H, Levy M, Cheng Q, Wang T and Remais JV: Transport and public health in China: The road to a healthy future. Lancet 390: 1781-1791, 2017.

7. Katz NP, Paillard FC and Ekman E: Determining the clinical importance of treatment benefits for interventions for painful orthopedic conditions. J Orthop Surg Res 10: 24, 2015.

8. Shao J, Chang H, Zhu Y, Chen W, Zheng Z, Zhang H and Zhang Y: Incidence and risk factors for surgical site infection after open reduction and internal fixation of tibial plateau fracture: A systematic review and meta-analysis. Int J Surg 41 176-182, 2017.

9. Richards MJ, Edwards JR, Culver DH and Gaynes RP: Nosocomial infections in medical intensive care units in the United States. National nosocomial infections surveillance system. Crit Care Med 27: 887-892, 1999.

10. Gheorghe A, Moran G, Duffy H, Roberts T, Pinkney T and Calvert M: Health utility values associated with surgical site infection: A systematic review. Value Health 18: 1126-1137, 2015.

11. Triantafyllopoulos G, Stundner O, Memtsoudis S and Poultsides LA: Patient, surgery, and hospital related risk factors for surgical site infections following total hip arthroplasty. ScientificWorldJournal 2015: 979560, 2015.

12. Gallo J, Holinka M and Moucha CS: Antibacterial surface treatment for orthopaedic implants. Int J Mol Sci 15: 13849-13880, 2014.

13. Trampuz A, Salzmann S, Antheaume J and Daniels AU: Microcalorimetry: A novel method for detection of microbia contamination in platelet products. Transfusion 47: 1643-1650, 2007.

14. De Vries FEE, Wallert ED, Solomkin JS, Allegranzi B, Egger M, Dellinger EP and Boermeester MA: A systematic review and meta-analysis including GRADE qualification of the risk of surgical site infections after prophylactic negative pressure wound therapy compared with conventional dressings in clean and contaminated surgery. Medicine (Baltimore) 95: e4673, 2016.

15. Mattiassich G, Ortmaier R, Rittenschober F and Hochreiter J: Diagnostic parameters in periprosthetic infections: The current state of the literature. Eur J Orthop Surg Traumatol 28 1573-1580, 2018.

16. Graves N, Wloch C, Wilson J, Barnett A, Sutton A, Cooper N, Merollini K, McCreanor V, Cheng Q, Burn E, et al: A cost-effectiveness modelling study of strategies to reduce risk of infection following primary hip replacement based on a systematic review. Health Technol Assess 20: 1-144, 2016.

17. Popa M, Cîrstoiu M, Cîrstoiu C, et al: Algic syndrome in osteoarticular infectious pathology; detection and rapid treatment of the causative agent using microcalorimetry. Filodiritto Editore-Proceedings, pp589-594, 2018.

18. Braissant O, Wirz D, Göpfert B and Daniels AU: Biomedical use of isothermal microcalorimeters. Sensors (Basel) 10: 9369-9383, 2010.

19. Zaharia DC, Iancu C, Steriade AT, Muntean AA, Balint O, Popa VT, Popa MI and Bogdan MA: MicroDSC study of staphylococcus epidermidis growth. BMC Microbiol 10: 322, 2010

20. Popa MG, Muntean A, Popa VT, Dragomirescu CC, Eremia I, Nica S, Popa MI and Cîrstoiu C: Microcalorimetric growth evaluation of Candida albicans in different conditions. Rom Biotechnol Lett 25: 2140-2147, 2020.

21. Uçkay I, Hoffmeyer P, Lew D and Pitted D Prevention of surgical site infections in orthopaedic surgery and bone trauma: State-of-the-art update. J Hosp Infect 84: 5-12, 2013.

22. Schwarz EM, Parvizi J, Gehrke T, Aiyer A, Battenberg A, Brown SA, Callaghan JJ, Citak M, Egol K, Garrigues GE, et al: 2018 International consensus meeting on musculoskeletal infection: Research priorities from the general assembly questions. J Orthop Res 37: 997-1006, 2019.

23. Rak M, Kavčlč M, Trebše R and CőR A: Detection of bacteria with molecular methods in prosthetic joint infection: Sonication fluid better than periprosthetic tissue. Acta Orthop 87: 339-345, 2016.
24. Scherping SC Jr. and Aaron AD: Orthopedic Infections. In: Essentials of Orthopedic Surgery. Wiesel SW and Delahay JN (ed) 3rd edition. Springer, pp84-105, 2007

25. Bori G, McNally MA and Athanasou N: Histopathology in periprosthetic joint infection: When will the morphomolecular diagnosis be a reality? Biomed Res Int 2018: 141270, 2018.

26. Cursaru A, Cretu B, Serban B, Lupu AG, Iacobescu G, Popa M, Cursaru R and Cirstoiu C: Mechanical safety study and antibiotic-loaded polymethylmethacrylate spacers threshold, manufactured intraoperatively in orthopaedic surgery. Mater Plast 57: 317-324, 2021.

27. Higuera-Guisset J, Rodríguez-Viejo J, Chacón M, Muñoz FJ, Vigués $\mathrm{N}$ and Mas J: Calorimetry of microbial growth using a thermopile based microreactor. Thermochim Acta 427: 187-191, 2005.

28. Calvet EJP: Calorimeter. In. Edited by Patent US, vol. 3059471 , G01N25/48 ed. United States: Calvet, Edouard J.P., 1962. https:// patents.google.com/patent/US3059471A/en.

29. Calvet $\mathrm{E}$ and Prat $\mathrm{H}$ : Recent Progress in Microcalorimetry. Macmillan, New York, NY, 1963.

30. Chen HL, Yao J, Wang L, Wang F, Bramanti E, Maskow T and Zaray G: Evaluation of solvent tolerance of microorganisms by microcalorimetry. Chemosphere 74: 1407-1411, 2009.

31. Zaharia DC, Muntean A,Popa MG, Steriade AT, Balint O, MicutR, Iftene C, Tofolean I, Popa VT, Baicus C, et al: Comparative analysis of Staphylococcus aureus and Escherichia coli microcalorimetric growth. BMC Microbiol 13: 171, 2013.

32. Wadsö L: Isothermal microcalorimetry. Current problems and prospects. J Therm Anal Calorim 64: 75-84, 2001.

33. Wadsö I: Isothermal microcalorimetry in applied biology. Thermochim Acta 394: 305-311, 2002.

34. Braissant O, Keiser J, Meister I, Bachmann A, Wirz D, Göpfert B, Bonkat $\mathrm{G}$ and Wadsö I: Isothermal microcalorimetry accurately detects bacteria, tumorous microtissues, and parasitic worms in a label-free well-plate assay. Biotechnol J 10: 460-468, 2015.

35. Borriello SP, Murray PR and Funke G (eds): Topley and Wilson's Microbiology and Microbial Infections. Bacteriology. Vol. 1. 10th edition. Hodder Arnold, London, 2005.

36. Madigan MT: Brock Biology of Microorganisms. Benjamin Cummings, San Francisco, CA, 2012.

37. Kong W, Wang J, Xing X, Jin C, Xiao X, Zhao Y, Zhang P, Zang Q and Li Z: Screening for novel antibacterial agents based on the activities of compounds on metabolism of Escherichia coli: A microcalorimetric study. J Hazard Mater 185: 346-352, 2011.

38. R Core Team: R: A language and environment for statistical computing. R Foundation for Statistical Computing, Vienna, Austria, 2013. http://www.R-project.org/.

39. RStudio Team: RStudio: Integrated development for R. RStudio, PBC, Boston, MA, 2020. http://www.rstudio.com/.

40. Popa MI, Cursaru A, Popa TV, Muntean AA, Bogdan S, Crețu B, Iordache $\mathrm{S}$ and Cirstoiu C: Study of bacterial proliferation using a method that shows bacterial growth depending on the heat released-microcalorimetry. Proc Rom Acad Series B 23: 197-203, 2021.

41. Popa MIG, Cursaru A, Serban B, Cretu B, Muntean AA, Popa TV, Chifiriuc MC and Cirstoiu C: Microcalorimetry-versatile method of describing bacterial growth. Appl Sci 11,9740, 2021.

42. Braissant O, Wirz D, Gopfert B and Daniels AU: Use of isothermal microcalorimetry to monitor microbial activities. FEMS Microbiol Lett 303: 1-8, 2010.

43. Popa M, Popa V, Serban B, Nedelcu R, Cretu B, Cursaru A and Cîrstoiu C: Utility of microcalorimetru in describing the growth curve of Candida albicans at different temperatures-identitying the optimal growth temperature. Rom J Orthop Surg Traumatol 2: 69-74, 2019.

44. Knight R, Spoors LM, Costa ML and Dutton SJ: Wound healing in surgery for trauma (WHIST): Statistical analysis plan for a randomised controlled trial comparing standard wound management with negative pressure wound therapy. Trials 20 : 186,2019

45. Matthew L, Achten J, Knight J, Bruce J, Dutton SJ, Madan J, Dritsaki M, Parsons N, Fernandez M, Grant R, et al: Effect of incisional negative pressure wound therapy vs. standard wound dressing on deep surgical site infection after surgery for lower limb fractures associated with major trauma: The WHIST randomized clinical trial. JAMA 323: 519-526, 2020. 